\title{
Real-life practice in the management of new-onset postoperative atrial fibrillation early after cardiac surgery
}

\author{
Michelle Berresheim BSc(Pharm) ACPR ${ }^{1}$, Arden R Barry BSc BSc(Pharm) PharmD ACPR ${ }^{2}$, \\ Colleen M Norris RN BScN MScN PhD ${ }^{3,4}$, Sheri L Koshman BSc(Pharm) PharmD ACPR ${ }^{3,5}$, \\ Jayan Nagendran MD PhD FRCSC ${ }^{3,6}$, Glen J Pearson BSc BScPhm PharmD FCSHP ${ }^{3,5}$
}

\begin{abstract}
M Berresheim, AR Barry, CM Norris, SL Koshman, J Nagendran, GJ Pearson. Real-life practice in the management of new-onset postoperative atrial fibrillation early after cardiac surgery. Curr Res Cardiol 2015;2(2):73-76.
\end{abstract}

OBJECTIVES: To investigate the real-world pharmacological management of postoperative atrial fibrillation $(\mathrm{POAF})$ in patients undergoing

METHODS: A retrospective cohort analysis consisting of adult patients who underwent coronary artery bypass grafting, valve or combined surgery from January to December 2011 was performed using a clinical registry. The peri- and postoperative pharmacological management (rate control, rhythm control, anticoagulant therapy) of POAF was evaluated. Stepwise multivariate regression analyses were used to identify determinants for medication use at discharge.

RESULTS: The cohort consisted of 1145 patients, of whom 377 (32.9\%) developed POAF and 271 (23.7\%) were included. At discharge, and/or rhythm-control therapy. Forty-eight (17.7\%) patients received cardiac surgery. 251 patients $(92.6 \%)$ received $\beta$-blocker therapy and $122(45.0 \%)$ received antiarrhythmic therapy. Two hundred sixty-one (96.3\%) received rate-

warfarin on discharge, although 38 had an additional indication. Men and urgent inpatients were less likely to be discharged on warfarin. Among 145 patients discharged on antiarrhythmic and/or anticoagulant therapy, $121(83.4 \%$ ) attended follow-up. Only 28.1\% (34 of 121) had an electrocardiogram or Holter monitoring performed; despite this, antiarrhythmic medications were either continued or not addressed in $47.7 \%$ (51 of 107) of patients discharged on therapy.

CONCLUSIONS: Treatment of POAF with rate- and/or rhythm-control medications was consistent with current national guideline recommendations. However, anticoagulant therapy use was low and appeared to be limited to patients with another indication. Assessment of POAF medications and rhythm status at postoperative follow-up visit was inconsistent. Thus, efforts to improve the management of POAF should focus on appropriately discontinuing unnecessary medications at postoperative follow-up to minimize the risk of adverse effects.

Key Words: ACE inhibitors; Amiodarone; Antiarrhythmics; Anticoagulants; Atrial fibrillation; Beta-blockers; Coronary artery bypass graft surgery; Health outcomes research; Nondihydropyridine calcium channel blockers; Rate control; Rhythm control; Warfarin

Dostoperative atrial fibrillation (POAF) is a frequent complication 1 of cardiac surgery (1-3). Although the majority of new-onset cases are self-limiting, POAF has been associated with an increased risk for hemodynamic deterioration, thromboembolic events and cognitive impairment (3-6). It has also been shown to prolong length of hospital stay and increase health care costs $(1,2)$. The incidence of POAF after cardiac surgery ranges from $30 \%$ to $50 \%$ and varies according to the type of surgery (eg, coronary artery bypass grafting [CABG], valve replacement/repair), with the highest incidence occurring in patients who undergo concurrent CABG and valve surgeries $(3,7)$. Patient characteristics associated with an increased risk for developing POAF include advanced age ( $>70$ years), male sex, history of hypertension, history of preoperative atrial fibrillation $(\mathrm{AF})$, need for intraoperative balloon pump and prolonged postoperative ventilation (>24 h) (1-4).

Recent guidelines from the Canadian Cardiovascular Society recommend strategies for the prevention and treatment of POAF (8). These include controlling ventricular response rate with $\beta$-blockers, nondihydropyridine calcium channel blockers or amiodarone; preventing cardioembolic stroke with anticoagulant medications; and converting patients to normal sinus rhythm. Pharmacological therapy for maintenance of sinus rhythm is preferred over isolated direct current cardioversion in this setting due to the high risk for early AF recurrence (8).

The Mazankowski Alberta Heart Institute (MAHI) is a quaternary care referral centre in Edmonton, Alberta, where approximately 1200 cardiac surgeries are performed annually. The local practice patterns and concordance with national guidelines at our institution are currently unknown. Optimal application of evidence-based pharmacotherapy and appropriate follow-up are essential to minimize the risks associated with treatment. The objective of the present study was to compare the pharmacological management of POAF in cardiac surgery patients at our institution with national guidelines, and identify determinants for use of rate-control, rhythm-control and anticoagulant medications.

\section{METHODS}

A single-centre retrospective cohort analysis was performed. The cohort consisted of consecutive patients who underwent cardiac surgery at the MAHI over a 12-month period (January 1 to December 31, 2011). Data regarding the peri- and postoperative management of patients who developed POAF were collected using the Alberta Provincial Project for Outcome Assessment in Coronary Heart Disease (APPROACH) database, a clinical registry that prospectively collects information on all patients who undergo cardiac surgery in Alberta (9). Follow-up data on patients discharged from hospital on an antiarrhythmic and/or anticoagulant medication were collected from outpatient records. Included were patients $\geq 18$ years of age who had undergone $\mathrm{CABG}$ and/or a valve replacement/repair surgery and developed new-onset POAF. The prespecified definition of POAF was the occurrence of AF requiring treatment (eg, pharmacological therapy, direct current cardioversion) post-cardiac surgery in patients who did not have preoperative AF. Patients with a history of a preoperative atrial or ventricular arrhythmia, atrial ablation, Cox maze procedure, or who had a cardiac pacemaker or automated implantable cardioverter

${ }^{1}$ Pharmacy Services, Alberta Health Services, Grande Prairie; ${ }^{2}$ Pharmacy Services, Alberta Health Services, Edmonton; ${ }^{3}$ Faculty of Medicine; ${ }^{4}$ School of

Public Health, University of Alberta; ${ }^{5}$ Department of Medicine; ${ }^{6}$ Department of Surgery, Mazankowski Alberta Heart Institute, Edmonton, Alberta

Correspondence: Dr Glen J Pearson, Suite 2C2, WMC, University of Alberta, 8440 - 112th Street, Edmonton, Alberta T6G $2 B 7$.

Telephone 780-407-2044,fax 780-407-6452,e-mail glen.pearson@ualberta.ca 
TABLE 1

Baseline cohort characteristics $(n=271)$

\begin{tabular}{|c|c|}
\hline Age, years, mean \pm SD & $68.3 \pm 9.7$ \\
\hline Male sex & $217(80.1)$ \\
\hline Postoperative length of stay, days, mean \pm SD & $9.1 \pm 4.8$ \\
\hline \multicolumn{2}{|l|}{ Comorbidities } \\
\hline Hypertension & $229(84.5)$ \\
\hline Previous myocardial infarction & $107(39.5)$ \\
\hline Chronic obstructive pulmonary disease & $106(39.1)$ \\
\hline Heart failure & $40(14.8)$ \\
\hline Previous percutaneous coronary intervention & $37(13.7)$ \\
\hline Previous coronary artery bypass graft & $6(2.2)$ \\
\hline \multicolumn{2}{|l|}{ Extent of coronary artery disease } \\
\hline Three-vessel disease & $100(36.9)$ \\
\hline Left main disease & $73(26.9)$ \\
\hline One- or two-vessel disease & $38(14.0)$ \\
\hline Normal/data missing & $60(22.1)$ \\
\hline \multicolumn{2}{|l|}{ Left ventricular ejection fraction } \\
\hline$\geq 35 \%$ & $104(38.4)$ \\
\hline$<35 \%$ & $11(4.1)$ \\
\hline Data missing & $156(57.6)$ \\
\hline \multicolumn{2}{|l|}{ Preoperative medications } \\
\hline Acetylsalicylic acid & $151(55.7)$ \\
\hline$\beta$-blocker & $188(69.4)$ \\
\hline Angiotensin-converting enzyme inhibitor & $104(38.4)$ \\
\hline Thienopyridine (clopidogrel or ticlopidine) & $27(10.0)$ \\
\hline Warfarin & $1(0.4)$ \\
\hline \multicolumn{2}{|l|}{ Type of surgery } \\
\hline Coronary artery bypass graft & $156(57.6)$ \\
\hline Valve & $65(24.0)$ \\
\hline Combined coronary artery bypass graft and valve & $50(18.4)$ \\
\hline \multicolumn{2}{|l|}{ Valve procedure performed ${ }^{*}$} \\
\hline Aortic valve repair & $5(1.8)$ \\
\hline Aortic valve replacement (bioprosthetic) & $75(27.7)$ \\
\hline Aortic valve replacement (mechanical) & $8(3.0)$ \\
\hline Mitral valve repair & $18(6.6)$ \\
\hline Mitral valve replacement (bioprosthetic) & $12(4.4)$ \\
\hline Mitral valve replacement (mechanical) & $0(0.0)$ \\
\hline \multicolumn{2}{|l|}{ Priority of surgery } \\
\hline Low risk & $22(8.1)$ \\
\hline Urgent outpatient & $154(56.8)$ \\
\hline Urgent inpatient & $85(31.4)$ \\
\hline Emergency & $10(3.7)$ \\
\hline
\end{tabular}

Data presented as $n(\%)$ unless otherwise indicated. ${ }^{*}$ Three patients under went $>1$ valve procedure

defibrillator were excluded. As well, patients taking antiarrhythmic medications on admission or who had a postoperative length of stay $>28$ days or died during their index hospitalization were also excluded. The present study was approved by the Health Research Ethics Board at the University of Alberta (Edmonton, Alberta).

The primary outcome was the proportion of patients discharged from hospital on $\beta$-blocker therapy. Prespecified secondary outcomes included percentage of patients discharged on an antiarrhythmic or anticoagulant medication, percentage of patients discharged on a rateversus rhythm-control strategy, determinants of a rate-control, rhythm-control or anticoagulant therapy at discharge, percentage of patients who continued on an antiarrhythmic or anticoagulant medication at postoperative follow-up and percentage of patients who had an objective measure of heart rhythm (eg, electrocardiogram, Holter monitor) at follow-up.

Data analysis was completed using SPSS version 21.0 (IBM Corporation, USA). Descriptive statistics were used for baseline
TABLE 2

Pharmacological therapy on discharge $(n=271)$

\begin{tabular}{lc}
\hline$\beta$-blocker therapy & $251(92.6)$ \\
Preoperative $\beta$-blocker continued & $178(65.7)$ \\
$\beta$-blocker initiated perioperatively & $73(26.9)$ \\
Antiarrhythmic therapy & $122(45.0)$ \\
Amiodarone & $121(44.6)$ \\
Other & $1(0.4)$ \\
Rate- and/or rhythm-control therapy & $261(96.3)$ \\
Rate- and rhythm-control therapy & $114(42.1)$ \\
Anticoagulant therapy (warfarin) & $48(17.7)$ \\
\hline Data presented as $n$ (\%)
\end{tabular}

TABLE 3

Determinants for use of anticoagulant medication at discharge

\begin{tabular}{lccc}
\hline Variable & OR & 95\% Cl & P \\
\hline Male sex & 0.33 & $0.13-0.89$ & 0.028 \\
Urgent inpatient surgery & 0.14 & $0.03-0.72$ & 0.019 \\
Valvular surgery & 8.80 & $1.20-64.40$ & 0.032 \\
Combined CABG and valve surgery & 6.98 & $2.03-24.02$ & 0.002 \\
\hline
\end{tabular}

CABG Coronary artery bypass graft

demographic and clinical characteristics, as well as the proportional primary and secondary outcomes. A stepwise multivariate logistic regression was completed to identify determinants for use of ratecontrol, rhythm-control and anticoagulant medications at discharge. Each analysis was adjusted for age, sex and any variable that demonstrated significant predictive value $(\mathrm{P}<0.10)$ for the dependent outcome based on the univariate analysis. $\mathrm{P}<0.05$ was considered to be statistically significant.

\section{RESULTS}

The cohort consisted of 1145 patients. Of these patients, 377 (32.9\%) developed POAF and 271 (23.7\%) met the inclusion criteria. The most common reasons for exclusion included a preoperative arrhythmia or antiarrhythmic medication (81 of 106 [76.4\%]), postoperative length of stay $>28$ days (26 of 106 [24.5\%]) and mortality during index hospitalization (15 of 106 [14.2\%]). Patient baseline demographic and clinical characteristics are included in Table 1.

The primary outcome occurred in $92.6 \%$ (251 of 271) of patients (Table 2). One hundred twenty-two patients (122 of 271 [45.0\%]) were discharged on an antiarrhythmic medication and 48 (48 of 271 [17.7\%]) were discharged on an anticoagulant medication (warfarin). Two hundred sixty-one patients (261 of 271 [96.3\%]) were discharged on either rate- or rhythm-control therapy. One hundred thirty-seven (137 of 271 [50.6\%]) were discharged on a ratecontrol strategy only ( $\beta$-blocker or nondihydropyridine calcium channel blockers) and 10 patients (10 of 271 [3.7\%]) were discharged on a rhythm-control strategy only. One hundred fourteen patients (114 of 271 [42.1\%]) were discharged on both a rate- and rhythmcontrol agent.

The multivariate analysis identified no independent predictors for use of rate- or rhythm-control medications at discharge. However, male sex, urgent inpatient surgery, isolated valve surgery and combined CABG/valve surgery were independent predictors for use of an anticoagulant medication at discharge (Table 3).

One hundred forty-five (145 of 271 [53.5\%]) patients were discharged on an antiarrhythmic and/or anticoagulant medication (Table 4). Only 121 (121 of 145 [83.4\%]) attended postoperative follow-up with a cardiac surgeon (mean 59 days postoperatively). Of these patients, 107 patients (107 of 121 [88.4\%]) were discharged on an antiarrhythmic medication (amiodarone). In 56 patients (56 of 107 [52.3\%]), amiodarone was discontinued either 
before or at follow-up. Amiodarone was continued in 18 patients (18 of 107 [16.8\%]) and not assessed in 33 patients (33 of 107 [30.8\%]) at follow-up. Only 34 patients that attended follow-up (34 of 121 [28.1\%]) had an electrocardiogram or Holter monitoring performed at the follow-up appointment. Thirty-seven patients that attended follow-up (37 of 121 [30.6\%]) were discharged on warfarin, which was continued in 23 patients (23 of 37 [62.2\%]) and discontinued in eight of $37(21.6 \%)$ patients. Warfarin was not addressed in six of $37(16.2 \%)$ patients at follow-up.

\section{DISCUSSION}

POAF is a common complication of cardiac surgery and has been associated with adverse patient outcomes (1-6). The annual incidence of newonset $\mathrm{POAF}$ in patients who underwent $\mathrm{CABG}$ and/or valve surgery at the MAHI in 2011 was approximately 33\%, which is consistent with the published literature $(3,7)$. Data regarding risk factors for POAF were not collected because they are already well defined in the literature (1-4).

While POAF is frequently self-limiting, the goals of therapy are parallel to those for AF in other clinical settings. In the absence of conclusive evidence comparing a rate- versus rhythm-control strategy in patients with POAF, guidelines suggest that either may be appropriate (8). It is also recommended that anticoagulation be considered in patients who remain in AF for $>72 \mathrm{~h}$. The use of rate- and rhythmcontrol medications for the treatment of POAF at the MAHI was predominantly consistent with current national guidelines; approximately $96 \%$ of patients received a rate-control and/or antiarrhythmic medication. However, surprisingly few patients (48 of 271 [17.7\%]) were recommended warfarin therapy on discharge, the majority of whom (38 of 48 [79.2\%]) had another compelling indication (eg, mechanical valve or mitral valve repair/replacement). The reason for the low utilization of warfarin on discharge is not clear; however, it may be reflective of most patients having a short duration of $\mathrm{AF}(<72 \mathrm{~h})$ or concern regarding increased risk for bleeding in the early postoperative period.

Multivariate regression analyses did not identify any independent determinants for use of rate- or rhythm-control medications at discharge, which may be the result of the small sample size. However, multiple determinants for anticoagulant therapy were identified. Men were less likely to be discharged on warfarin, which may be secondary to the small number of women included in the cohort (approximately $20 \%$ ). Patients who underwent urgent inpatient surgery were also less likely to be on warfarin at discharge. This may be related to the increased acuity and comorbid disease burden associated with urgent surgical candidates, which often equates to an increased risk for bleeding. Patients who underwent any type of valvular surgery were more likely to be discharged on warfarin compared with patients who underwent isolated CABG surgery; this likely demonstrates the appropriate use of anticoagulant therapy in patients with valvular heart disease.

In most patients, POAF will resolve within six to 12 weeks postoperatively $(10,11)$. Accordingly, medications for POAF should be recommended for a defined period of time or reassessed at postoperative follow-up to eliminate unnecessary medications and minimize the risk for adverse effects $(8,12-14)$. In this cohort, postoperative followup was lower than expected (approximately $83 \%$ ), but was within the recommended timeframe (mean 8.4 weeks) (8). Warfarin was discontinued in only one-fifth of patients discharged on therapy because most patients had an additional indication for prolonged treatment (eg, mechanical valve, mitral valve replacement/repair). Unexpectedly, less than one-third of patients received an objective measure of heart rhythm assessment at follow-up, despite a high percentage of patients being discharged on a rhythm-control medication. Regardless, antiarrhythmic therapy was either continued or not addressed in almost one-half of patients. While a rhythm-control strategy is recommended in the immediate postoperative period to prevent early recurrence, converting to a rate-control strategy is typically preferred for patients who remain in AF six to 12 weeks postoperatively due to a lack of benefit and increased adverse effects with antiarrhythmics compared with negative chronotropic agents $(8,13,15,16)$.

\section{TABLE 4}

Follow-up cohort baseline characteristics $(n=145)$

\begin{tabular}{|c|c|}
\hline Age, years, mean \pm SD & $67.4 \pm 9.6$ \\
\hline Male sex & $116(80.0)$ \\
\hline Postoperative length of stay, days, mean \pm SD & $8.9 \pm 4.3$ \\
\hline \multicolumn{2}{|l|}{ Comorbidities } \\
\hline Hypertension & $122(84.1)$ \\
\hline Previous myocardial infarction & $52(35.9)$ \\
\hline Chronic obstructive pulmonary disease & $24(16.6)$ \\
\hline Heart failure & $3(2.1)$ \\
\hline Previous percutaneous coronary intervention & $26(17.9)$ \\
\hline Previous coronary artery bypass graft & $58(40.0)$ \\
\hline \multicolumn{2}{|l|}{ Extent of coronary artery disease } \\
\hline Three-vessel disease & $53(36.6)$ \\
\hline Left main disease & $30(20.7)$ \\
\hline One- or two-vessel disease & $30(20.7)$ \\
\hline Normal/data missing & $32(22.1)$ \\
\hline \multicolumn{2}{|l|}{ Left ventricular ejection fraction } \\
\hline$\geq 35 \%$ & $52(35.9)$ \\
\hline$<35 \%$ & $3(2.1)$ \\
\hline Data missing & $90(62.0)$ \\
\hline \multicolumn{2}{|l|}{ Preoperative medications } \\
\hline Acetylsalicylic acid & $75(51.7)$ \\
\hline$\beta$-blocker & $94(64.8)$ \\
\hline Angiotensin-converting enzyme inhibitor & $49(33.8)$ \\
\hline Thienopyridine (clopidogrel or ticlopidine) & $17(11.7)$ \\
\hline Warfarin & $1(0.7)$ \\
\hline \multicolumn{2}{|l|}{ Type of surgery } \\
\hline Coronary artery bypass graft & $71(49.0)$ \\
\hline Valve & $40(27.6)$ \\
\hline Combined coronary artery bypass graft and valve & $34(23.4)$ \\
\hline \multicolumn{2}{|l|}{ Valve procedure performed* } \\
\hline Aortic valve repair & $5(3.4)$ \\
\hline Aortic valve replacement (bioprosthetic) & $39(26.9)$ \\
\hline Aortic valve replacement (mechanical) & $8(5.5)$ \\
\hline Mitral valve repair & $15(10.3)$ \\
\hline Mitral valve replacement (bioprosthetic) & $9(6.2)$ \\
\hline Mitral valve replacement (mechanical) & $0(0.0)$ \\
\hline \multicolumn{2}{|l|}{ Priority of surgery } \\
\hline Low risk & $14(9.7)$ \\
\hline Urgent outpatient & $88(60.7)$ \\
\hline Urgent inpatient & $34(23.4)$ \\
\hline Emergency & $9(6.2)$ \\
\hline
\end{tabular}

$\overline{\text { Data presented as } n(\%) \text { unless otherwise indicated. }{ }^{*} T w o \text { patients underwent }}$ $>1$ valve procedure

The present study has several limitations that warrant discussion. Although data in the APPROACH registry are collected prospectively, the registry is limited by a finite number of characteristics and outcome measures. The perioperative sequence of events was not captured, making it difficult to assess the proportion of patients who experienced additional complications secondary to POAF and whether more than one treatment intervention was required. The registry captures medications at the time of admission and discharge, but does not include inpatient medication use. As a result, it was not possible to evaluate how POAF therapy was selected, and the temporal relationship between the development of POAF and initiation of pharmacotherapy. The registry also does not capture whether patients remained in $\mathrm{AF}$ for $>72 \mathrm{~h}$; thus, it was not possible to evaluate the appropriateness of anticoagulant medications at discharge. Moreover, patients who had an indication other than POAF for long-term anticoagulation were not differentiated in the analysis. Follow-up data were limited by the completeness of documentation in the outpatient records. 
In addition, follow-up from other providers (eg, cardiologists, general practitioners) was not consistently documented.

\section{CONCLUSIONS}

Initiation of rate- and rhythm-control medications at discharge for the treatment of POAF in cardiac surgery patients at the MAHI was consistent with current national guideline recommendations. However, the use of anticoagulant therapy on discharge was low and appeared to be limited to patients with another indication (eg, mechanical valve, mitral valve replacement/repair). Assessment of POAF medications and rhythm status at the six- to 12-week postoperative follow-up visit was inconsistent. Antiarrhythmic medication was either continued or not addressed in almost one-half

\section{REFERENCES}

1. Aranki SF, Shaw DP, Adams DH, et al. Predictors of atrial fibrillation after coronary artery surgery. Current trends and impact on hospital resources. Circulation 1996;94:390-7.

2. Mathew JP, Parks R, Savino JS, et al. Atrial fibrillation following coronary artery bypass graft surgery: Predictors, outcomes, and resource utilization. MultiCenter Study of Perioperative Ischemia Research Group. JAMA 1996;276:300-6.

3. Creswell LL, Schuessler RB, Rosenbloom M, Cox JL. Hazards of postoperative atrial arrhythmias. Ann Thorac Surg 1993;56:539-49.

4. Ommen SR, Odell JA, Stanton MS. Atrial arrhythmias after cardiothoracic surgery. N Engl J Med 1997;336:1429-34.

5. Villareal RP, Hariharan R, Liu BC, et al. Postoperative atrial fibrillation and mortality after coronary artery bypass surgery. J Am Coll Cardiol 2004;43:742-8.

6. Stanley TO, Mackensen GB, Grocott HP, et al. The impact of post-operative atrial fibrillation on neurocognitive outcome after coronary artery bypass graft surgery. Anesth Analg 2002;94:290-5.

7. Mitchell LB. Incidence, timing and outcome of atrial tachyarrhythmias after cardiac surgery. In: Steinberg JS, ed. Atrial Fibrillation After Cardiac Surgery. Norwell: Kluwer Academic Publishers, 2000:37-50.

8. Mitchell LB, CCS Atrial Fibrillation Guidelines Committee. Canadian Cardiovascular Society Atrial Fibrillation Guidelines 2010: Prevention and treatment of atrial fibrillation following cardiac surgery. Can J Cardiol 2011;27:91-7. of the cohort, and less than one-third of patients underwent an objective investigation of rhythm status. Thus, efforts to improve the management of POAF should focus on a systematic approach to the assessment and appropriate discontinuation of unnecessary medications at postoperative follow-up to minimize the risk for adverse effects.

ACKNOWLEDGEMENTS: The authors thank Mary Ann James from APPROACH for her assistance with data extraction.

DISCLOSURES: The authors have no real or potential conflicts of interest to disclose.

9. Ghali WA, Knudtson ML. Overview of the Alberta Provincial Project for Outcome Assessment in Coronary Heart Disease. On behalf of the APPROACH investigators. Can J Cardiol 2000;16:1225-30.

10. Maisel WH, Rawn JD, Stevenson WG. Atrial fibrillation after cardiac surgery. Ann Intern Med 2001;135:1061-73.

11. Lee JK, Klein GJ, Krahn AD, et al. Rate-control versus conversion strategy in postoperative atrial fibrillation: A prospective, randomized pilot study. Am Heart J 2000;140:871-7.

12. Omae T, Kanmura Y. Management of postoperative atrial fibrillation. J Anesth 2012;26:429-37.

13. Wyse DG, Waldo AL, DiMarco JP, et al. A comparison of rate control and rhythm control in patients with atrial fibrillation. N Engl J Med 2002;347:1825-33.

14. Meurin P, Weber H, Renaud N, et al. Evolution of the postoperative pericardial effusion after day 15: The problem of the late tamponade. Chest 2004;125:2182-7.

15. Gillis AM, Verma A, Talajic M, et al. Canadian Cardiovascular Society Atrial Fibrillation Guidelines 2010: Rate and rhythm management. Can J Cardiol 2011;27:47-59.

16. Testa L, Biondi-Zoccai GG, Dello Russo A, et al. Rate-control vs. rhythm control in patients with atrial fibrillation: A meta-analysis. Eur Heart J 2005;26:2000-6. 